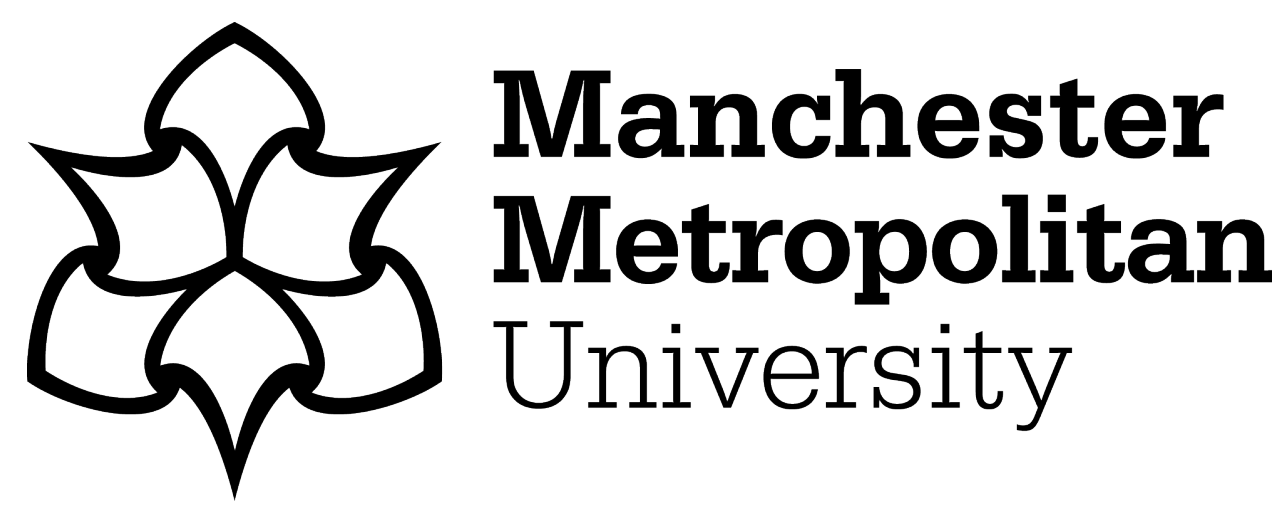

Southgate, MJ, Taylor, CT, Hutchinson, S and Bowring, NJ (2014) Faster with CLEAN - An exploration of the effects of applying a nonlinear deconvolution method to a novel radiation mapper. In: SPIE Security + Defence, 22 September 2014 - 25 September 2014, Amsterdam, Netherlands.

Downloaded from: https://e-space.mmu.ac.uk/621319/

Version: Accepted Version

Publisher: Society of Photo-Optical Instrumentation Engineers (SPIE) DOI: https://doi.org/10.1117/12.2067573

Please cite the published version 


\title{
Faster with CLEAN - An exploration of the effects of applying a non- linear deconvolution method to a novel radiation mapper
}

\author{
Matthew J. Southgate*a, Christopher T. Taylorb ${ }^{\mathrm{b}}$, Simon Hutchinson ${ }^{\mathrm{a}}$, Nicholas J. Bowring ${ }^{\mathrm{a}}$ \\ ${ }^{a}$ Centre for Sensing and Imaging, Manchester Metropolitan University, Chester Street, Manchester, \\ M1 5GD, UK; ' School of Physics and Astronomy, The University of Manchester, Oxford Road, \\ Manchester, M13 9PL, UK;
}

\begin{abstract}
This paper examines the suitability and potential of reducing the acquisition requirements of a novel radiation mapper through the application of the non-linear deconvolution technique, CLEAN. The radiation mapper generates a threshold image of the target scene, at a user defined distance, using a single pixel detector manually scanned across the scene . This paper provides a discussion of the factors involved and merits of incorporating CLEAN into the system. In this paper we describe the modifications to the system for the generation of an intensity map and the relationship between resolution and acquisition time for a target scene. The factors influencing image fidelity for a scene are identified and discussed with the impact on fill-factor of the intensity image, which in turn determines the ability of the operator to accurately identify features of the radiation source within a target scene. The CLEAN algorithm and its variants have been extensively developed by the radio astronomy community to improve the image fidelity of data collected by sparse interferometric arrays. However, the algorithm has demonstrated surprising adaptability including terrestrial imagery, as detailed in Taylor et al. SPIE 9078-19 and Bose et al., IEEE 2002. CLEAN can be applied directly to raw data via a bespoke algorithm. However, this investigation is a proof-of-concept and thus requires a well tested verification method. We have opted to use the public ally available implementation of CLEAN found in the Common Astronomy Software Applications (CASA) package. The use of CASA for this purpose dictates the use of simulated input data and radio astronomy standard parameters. Finally, this paper presents the results of applying CLEAN to our simulated target scene, with a discussion of the potential merits a bespoke implementation would yield.
\end{abstract}

Keywords: CLEAN, mm-wave, mapping, beam width measurement, low cost, antenna design verification.

\section{INTRODUCTION}

This paper describes the continued development of a manually scanned single pixel mm-wave imager. The improvements detailed in the paper relate to measurement speed, spatial resolution and the effects of post processing with a non-linear deconvolution CLEAN algorithm. Post-processing with CLEAN is investigated as a possible means of reducing the measurement requirements.

Numerous applications exist requiring the measurement and visualisation of radiation sources outside the visible spectrum, including laboratory based experiments requiring the alignment of multiple mm-wave devices and spatial radiation measurements for antenna characterisation. Currently operating in the frequency band centred around $94 \mathrm{GHz}$ the prototype described in this paper was developed as a proof of concept to satisfy the needs of the research team for a tool to measure and assist in the development of concealed threat detection systems ${ }^{1},{ }^{2}$. However, unlike existing passive imaging systems which are typically very expensive, bulky and contain complex and possibly moving optics ${ }^{3}{ }^{5}$ the prototype tool was designed to be lightweight, low cost, intuitively operated and operate with a range of interchangeable sensing elements ${ }^{1}, 2$. Radiation measurements and intensity mapping are displayed in real time for a 3D space at high frame rates. At this stage the system is incapable of producing imagery in real time useful for concealed threat detection, however it does offer a previously unavailable diagnostic capability for complex imaging systems. This system enables

\footnotetext{
*E-mail: m.southgate@mmu.ac.uk
} 
accurate and repeatable radiation intensity measurements with minimal effort of radiation sources where it is impractical to move or test them under laboratory conditions.

This paper is organised as follows: Section 2 describes the hardware and software elements of the system, Section 3 describes the method and theory of operation for the system, Section 4 introduces interferometric imaging and the CLEAN algorithm, Section 5 discusses the results and system implications of applying CLEAN during post processing, and Section 6 summaries and concludes the paper.

\section{THE SYSTEM}

The system consists of three parts, the Primary Unit (PU) for measurement of and initial signal processing of radiation data, a high speed infrared (IR) wireless camera, and a computer with a Bluetooth module to store, process and present the data. Communications between the individual elements of the system is via Bluetooth.

The PU was designed to: interface with different front ends to enable the system to work at different frequency bands, to condition the detected signal for acquisition by a microcontroller, and apply basic digital signal processing before transmission to the computer. Two highly directional IR Light Emitting Diodes (LED) act as beacons to indicate the position of the PU. All of these tasks are performed continuously in real-time. The prototype uses a Monolithic microwave integrated circuit (MMIC) RF front end with embedded amplifier sensitive to radiation in the $75-110 \mathrm{GH}$ band.

The self-contained wireless IR camera is an unmodified Wii Remote Controller, developed by Nintendo as a low-cost games controller for the Wii console ${ }^{6}$. The Wii Remote contains embedded hardware and signal processing for the identification and acquisition of up to four IR sources, greatly simplifying the task of tracking the PU's IR beacons. Information about the number and position of IR sources is continuously sent to the Computer. The on-board IR Camera has a resolution of $1024 \times 768$. The use of the Camera is fundamental to this system enabling accurate and repeatable measurements to be made by manually scanning the PU for distance and position in the Field Of View (FOV) of the Camera.

The Computer is a typical specification workstation running a custom C\# application on the Microsoft Windows 7 operating system. The only other hardware required is Bluetooth adaptor. The application matches incoming data from the PU and Camera, and populates an updateable point cloud with the processed data. The point cloud data is visualised in real time during data collection to provide feedback to the operator. The application can be configured to obtain intensity data in a user defined plane with respect to the radiation source suitable for processing and evaluation of the CLEAN algorithm. More detailed information about the hardware and software can be found in reference ${ }^{2}$.

\section{METHOD AND THEORY OF OPERATION}

The system components and setup are shown in Figure 1.

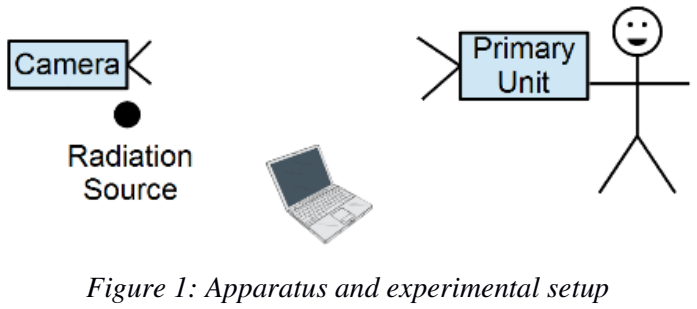

The PU is held in the hand, pointed at the radiation source to be measured and moved within the FOV of the Camera. The system will measure radiation values when the Camera is either unused or the PU is out of the FOV or alignment with the camera, however these values are normally discarded as a spatial position cannot be allocated to the measurement. For accurate spatial calculations the camera was calibrated with the PU. The calibration process determines the relationship between what appears on the image and the real-world position of the object. According to the rule of similar triangles the distance to the PU is calculated as illustrated in Figure 2 and Equation 1: 


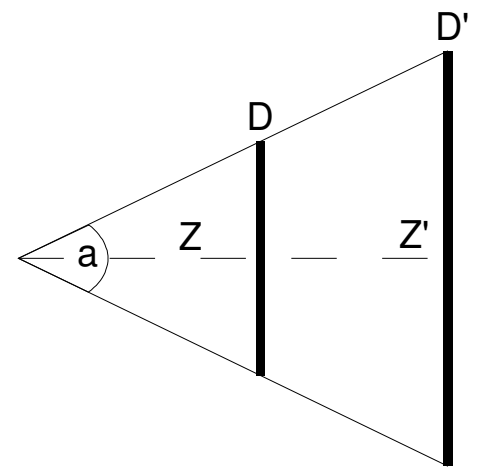

Figure 2: Similar triangles and the apparent size of an object in an image as a function of distance

$$
\frac{D}{Z}=\frac{D^{\prime}}{Z^{\prime}}
$$

where $D$ is a known dimension, $Z$ is a known distance from the camera and $a$ is a fixed angle - the FOV of the Camera. Therefore as the PU is moved away from the Camera, the PU appears smaller with the distance between the two beacons reducing as a function of distance to maintain the ratio. The application software uses an open-source library to interpret the output of the Camera ${ }^{7}$.

Tracking and localisation of the PU is achieved by processing the absolute and relative positions of the IR beacons as seen by the Camera. The use of two highly directional IR emitters on the sides of the PU mitigates the problem of misalignment of the PU within the FOV of the camera and the generation of a phantom depth. This also ensures the detector is pointing at the radiation source for an accurate measurement.

\subsection{System improvements}

Table 1: Table of specifications for image resolution and update rate for Mk1 and Mk2 systems

\begin{tabular}{|l|l|l|}
\hline Software Version & Mk1 & Mk2 \\
\hline Multithreading & No & Yes \\
\hline Image resolution & $256 \times 192$ & $1024 \times 768$ \\
\hline Update rate & $\begin{array}{l}30 \mathrm{~Hz} \text { dropping to } 8 \mathrm{~Hz} \text { over } 600 \\
\text { seconds }\end{array}$ & $100 \mathrm{~Hz}$ \\
\hline
\end{tabular}

To enable the investigation of CLEAN the application software was rewritten to fully utilise the capabilities of the Camera. To work effectively CLEAN requires an image of size greater that possible with the Mk1 software. However by increasing the resolution the time required to capture an image for the same fill factor of pixels increases and hence the need to multithread the application to operate at a maximum speed offsetting the increase in pixels. However, the time required to measure the radiation intensity in a plane is not a quick process to achieve a high fill factor.

\section{INTERFEROMETRIC IMAGING}

Interferometers have been developed by the radio astronomy community to facilitate high-resolution observations of far field target scenes through the use of a sparsely populated set of antenna elements. The detected signals from the collection of antennas are correlated in a pair-wise fashion, and each of these correlations measures a specific Fourier component of the target scene's brightness temperature / radiation power. The set of sampled Fourier components (known collectively as the Visibility function) populate the Fourier plane and by taking the Fourier Transform of this a reconstructed image of the target scene is produced (this is covered in greater detail in ${ }^{8}$ ). Due to the presence of 
unmeasured Fourier components, the finite extent of the array, as well as the introduction of system noise and other imaging artefacts, the reproduced image is an imperfect representation of the target scene. In radio astronomy terminology, this image is known as the dirty map. In effect, the dirty map is the perfect image of the target scene convolved with the Point Spread Function (PSF) of the antenna array (which contains significant sidelobes - the image plane portrayal of imperfect Fourier plane sampling).

\subsection{Non-linear deconvolution ${ }^{8}$}

The above indirect imaging method can be supplemented by various techniques to improve image fidelity. One such method developed by the radio astronomy community is the non-linear deconvolution technique known as CLEAN. This method operates on the data set after it has been transformed into the image plane (i.e. the dirty map), using estimations based on a priori knowledge of the target scene to readdress imperfections caused by the unmeasured regions of the Fourier plane. These assumptions include the entire image having positive intensity due to the emission of radiation and that the target scene being comprised of a distribution of uncorrelated point sources ${ }^{9}$.

CLEAN operates in an iterative loop, using the point of maximum correlation between the dirty map and PSF to determine the maximum peak in the data set. A user-defined percentage of the PSF is subtracted from the dirty map at this location to leave a residual image. A delta function is generated in a separate image with the same magnitude and location of the subtraction and is called a CLEAN component. This image plane delta function implicitly predicts a uniform distribution of data across the entire Fourier plane (as the Fourier transform of an image plane delta function is a constant throughout the Fourier plane). The residual image is fed back into the iterative process to replace the dirty map and is repeated until a stopping criteria is reached. From this, a set of CLEAN components are built up that describe the 'point sources' that the algorithm has detected and combine to produce a more realistic interpolation into the unmeasured Fourier regions. Figure 3 illustrates this part of the CLEAN algorithm with a simple example. Once the iterative process is complete, the CLEAN components are convolved with the PSF to enforce a degree of short-range coherence and finally the residual is added back in to create the final CLEAN image.
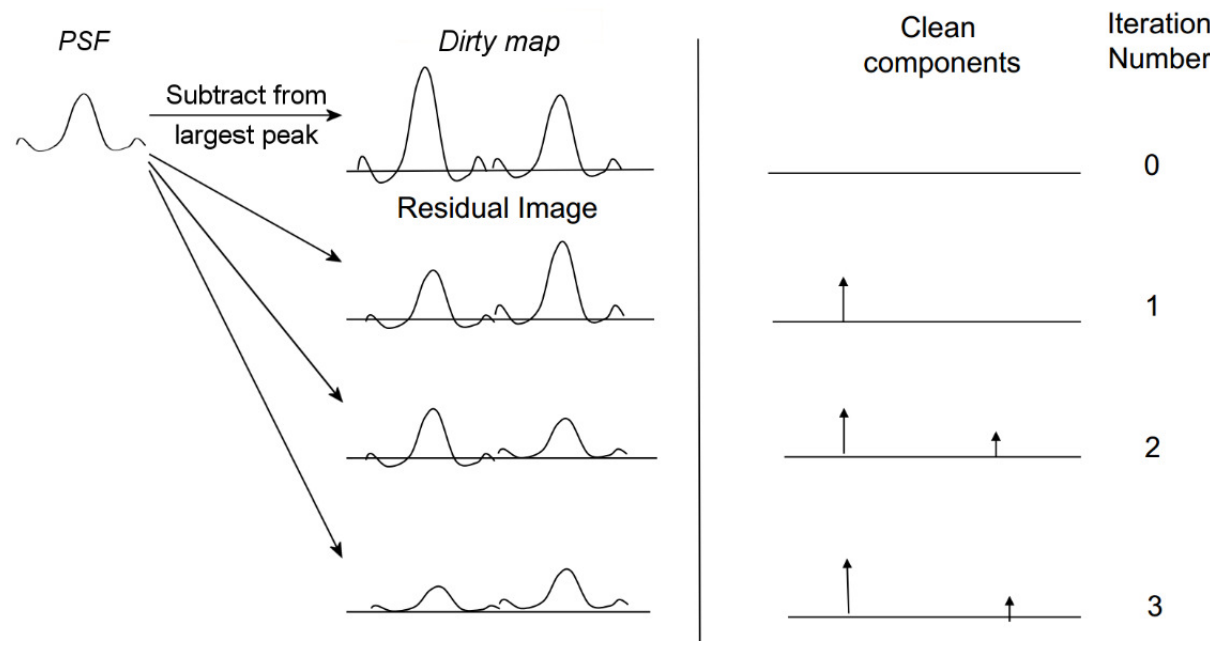

Figure 3: An illustration of the iterative process to generate CLEAN components. Note the decrease in peak intensity per iteration in the residual image is commensurate to the increase in CLEAN component intensity (image modified from J. Conway) ${ }^{8}$.

Despite its simplicity CLEAN has repeatedly proved its effectiveness on astronomical data sets covering a range of targets, including extended, smoothly distributed sources and those with relatively low radiometric temperature ranges. From the early success of the application of this technique to terrestrial targets ${ }^{8},{ }^{10}$, it was hoped that a similar improvement could be observed for application to imagery obtained by the radiation mapper. 


\section{RESULTS}

\subsection{Conversion of raw data}

The radiation source chosen to form the target scene was an active mm-wave concealed threat detection system. The radiation mapper PU was displaced through the FOV of the Camera to build up a raw data set with a fill factor of $89.84 \%$. The data set was converted into an 8-bit RGB image to be compatible with the CLEAN image processing technique (see Figure 4). The visual representation is characterised by a bright extended source surrounded by a uniform low-level background.

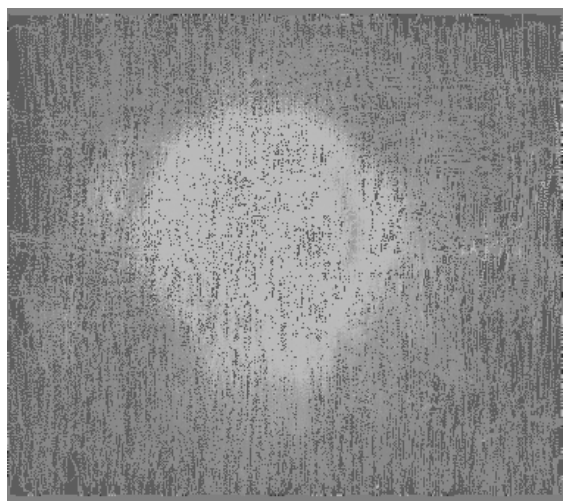

Figure 4: True-colour representation of the raw data after conversion into an 8-bit image for use as the target scene.

\subsection{Application of CLEAN}

For this initial exploration the authors chose to use the de-facto implementation of CLEAN used extensively by the radio astronomy community due to its outstanding reliability. In this implementation a simulated observation of the target scene (Figure 4) by a multi-element interferometer is generated, with the dirty map as the output. The CLEAN algorithm is then applied to the dirty map. Ideally, CLEAN would be applied directly to the target scene, but due to the constraints of the radio astronomy software package being utilised this is not possible. Therefore, this work can only be seen as an indication of the benefits of the application of CLEAN and not a definitive absolute result on any improvement obtained.

Figure 5 depicts the main characteristics of the observing interferometric array used in this simulation. It is important to stress that the choice of array configuration and use of radio astronomy standard parameters for this simulation, such as observing frequency, etc, are restrictions of the radio astronomy software package and are thus arbitrary.

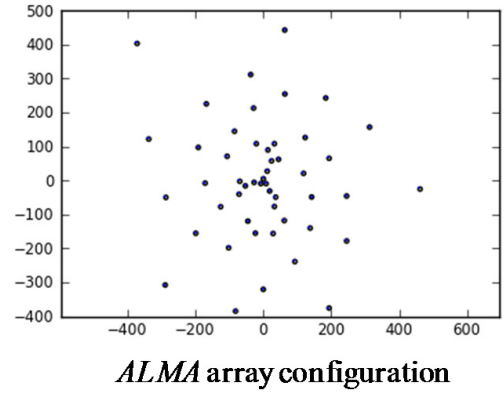

(50 antenna elements)

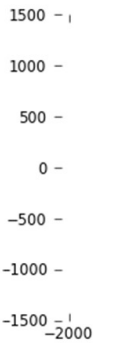

$-1500-1000$

$u, v$ coverage

(3 hour observation)

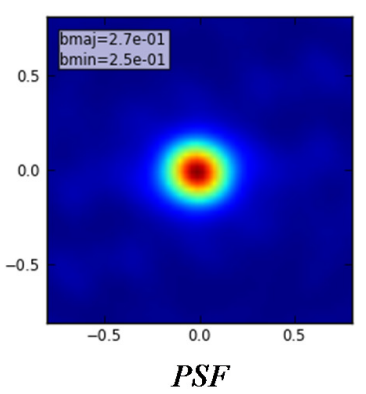

Figure 5: Characteristics of the simulated observing system within the radio astronomy software package. This is based on the Atacama Large Millimeter/Submillimeter Array (ALMA), and shows the physical antenna element positions (left), Fourier plane coverage (middle) and the PSF (right). 
The dirty map created by the simulated observation of the target scene is given in Figure 6 (left), next to a contrast enhanced version (right) to highlight the pixel intensity discrepancies. These variations directly relate to differences in the measured brightness temperature / radiation power and deviate from the uniform background of the target scene as shown in Figure 4. Note that the blurring of the dirty map due to the convolution between the target scene and the PSF has resulted in the insertion of data into a majority of the unmeasured regions when compared to the target scene. However, this blurring has distorted the shape of the target radiation source.
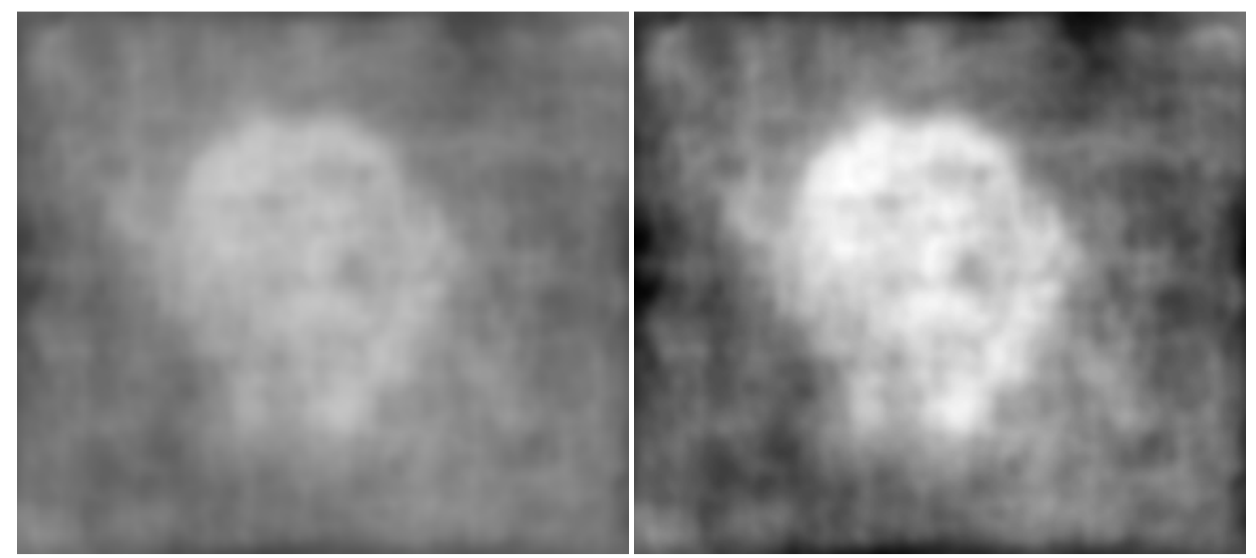

Figure 6:The resultant dirty map produced from a simulated observation of the target scene (left), and a contrast enhanced version (right) to highlight variations in pixel intensity.

The CLEAN algorithm was then applied to the dirty map and the final CLEAN image is reproduced in Figure 7 (left), with a contrast enhanced version (right). In comparison to the dirty map (Figure 6), the CLEAN image has a greater level of uniformity on a pixel-by-pixel basis. It can also be argued that the shape of the target radiation source is closer to the original target scene than the reconstructed dirty map.

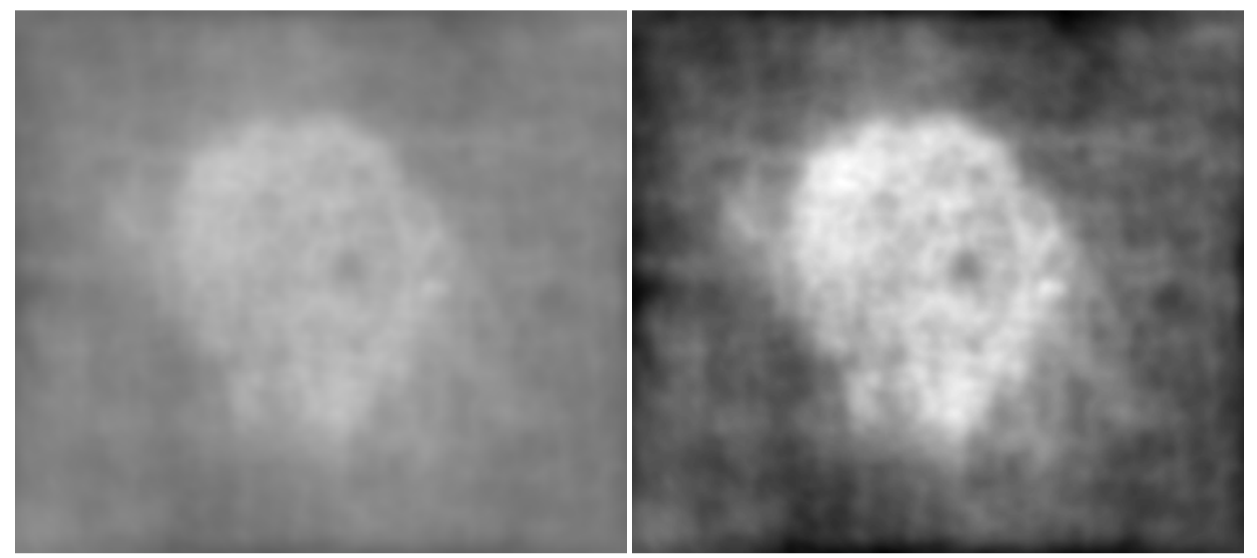

Figure 7: The CLEAN image (left) and its contrast enhanced counterpart (right). Note the smoothing of the background when compared to the dirty map (Figure 6).

For a mathematical treatment of the data sets difference maps of the original target scene with either the dirty map or CLEAN image were produced and the average percentage difference and the root mean squared (rms) variation between corresponding pixels was calculated. These results are presented in Table 2 and validate that the application of $C L E A N$ improves the image fidelity. The relatively small magnitude of this improvement can be attributed to the limitations imposed by the forced implementation of standard radio astronomy parameters. 
Table 2: The average percentage difference and rms variation in pixel intensity in difference maps generated from the simulated observation of the target scene with an $89.84 \%$ fill factor.

\begin{tabular}{|l|l|l|}
\hline Difference map & Average percentage difference (\%) & rms variation \\
\hline Target scene vs. dirty map & 8.23 & 26.53 \\
\hline Target scene vs. CLEAN & 7.05 & 24.48 \\
\hline
\end{tabular}

\subsection{Application of CLEAN with a reduced fill-factor}

The post-processing method outlined in Section 5.2 was repeated using a modified target scene with a 0.25 reduction in the fill factor to $67.38 \%$ (Figure 8). Applying the previous antenna element configuration (Figure 5) for a simulated observation of this reduced target scene produced the dirty map depicted in Figure 9. Note the increased pixel intensity fluctuations present throughout the image in comparison to the higher fill factor dirty map (Figure 6). The additional processing performed by the CLEAN algorithm attempts to adapt to the increase in unmeasured regions, however this increase led to a reduction in the visual clarity of the final image (Figure 10). Despite this perceived decrease, the difference map calculations presented in Table 3 illustrate that overall the CLEAN image is of higher image fidelity than the dirty map (albeit a smaller improvement than depicted in Section 5.2).

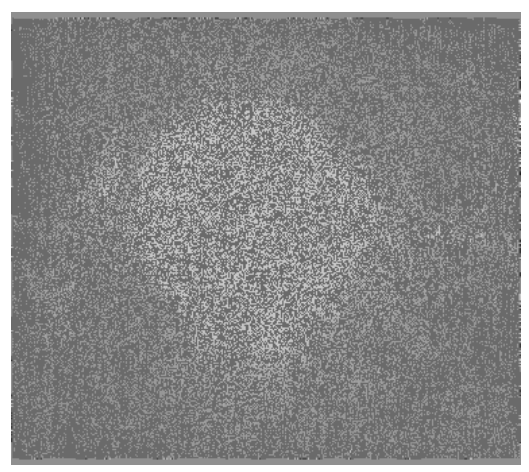

Figure 8: Target scene with a reduced fill factor $(67.38 \%)$. Note the degradation in image fidelity in comparison to the original target scene with a fill factor of $89.84 \%$ (Figure 4 ).
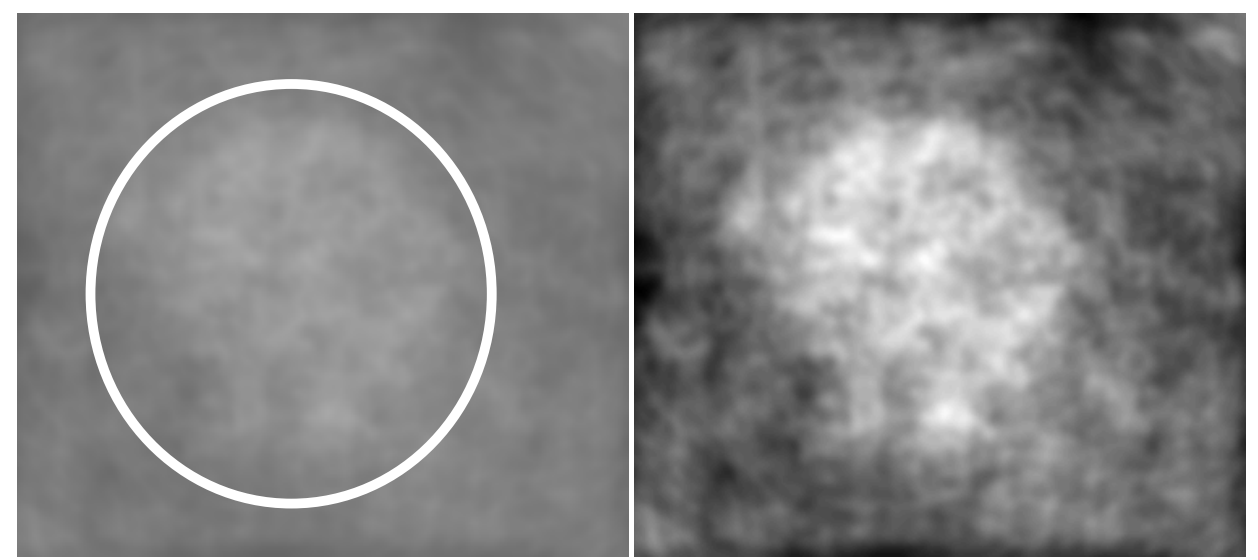

Figure 9: The dirty map produced from a reduced fill factor target scene (left) and its corresponding contrast enhanced version (right). Due to the poor contrast of the unenhanced dirty map, the approximate area of the radiation source has been highlighted. Note the increased pixel intensity fluctuations present throughout the image. 

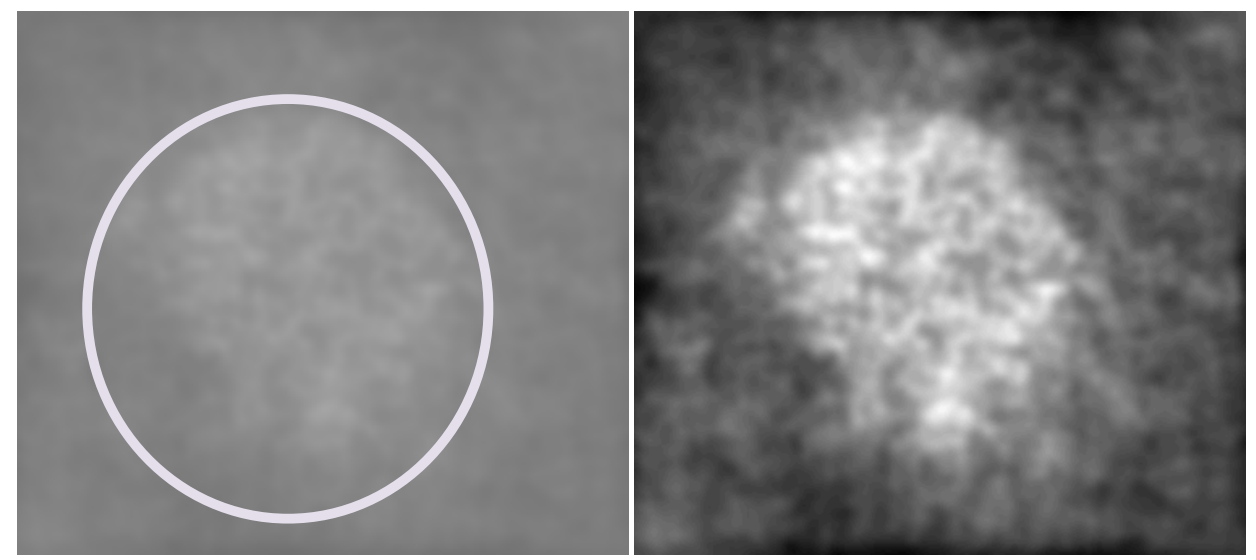

Figure 10:The CLEAN image (left) and a contrast enhanced adaptation (right) from the reduced fill factor simulation. Due to the poor contrast of the unenhanced dirty map, the approximate area of the radiation source has been highlighted.

Table 3:The average percentage difference and rms variation in pixel intensity for difference maps between images produced during the simulated observation of a target scene with fill factor $89.84 \%$ (top) and $67.38 \%$ (bottom).

\begin{tabular}{|c|l|l|}
\hline Difference map & Average percentage difference (\%) & rms variation \\
\hline Fill factor 89.84 \% & & \\
\hline Target scene vs. dirty map & 8.23 & 26.53 \\
\hline Target scene vs. CLEAN & 7.05 & 24.48 \\
\hline Fill factor 67.38\% & & \\
\hline Target scene vs. dirty map & 10.05 & 30.85 \\
\hline Target scene vs. CLEAN & 9.86 & 31.37 \\
\hline
\end{tabular}

The major conclusion to be drawn from Table 3 is that the application of CLEAN improves the image fidelity, and the magnitude of such an improvement is tied with the fill factor of the original target scene. Therefore the choice of fill factor still remains application specific; for the highest fidelity image possible a fill factor approaching $100 \%$ is still required, whereas an adequate level of fidelity for a given fill factor can be approached by applying CLEAN to a lower fill factor target scene.

This exploration warrants further investigation with the creation of a bespoke CLEAN algorithm that circumvents the radio astronomy software package and thus the dirty map stage of processing, alongside additional enhancements to the front-end hardware.

\section{SUMMARY}

The capabilities of a novel radiation mapper, capable of taking quick and repeatable measurements, have been enhanced in three key areas: resolution, speed and signal processing. The improvement in resolution enables more accurate spatial tracking of where radiation intensity measurements are taken resulting in better fidelity imagery. A better than three-fold improvement of measurement speed, achieved by rewriting the software for multithreading, has yielded multiple benefits including faster image capture times, reduced user fatigue, and overall a more responsive system and positive user experience. Lastly, the additional application of CLEAN to the raw data set has shown promising results in improving the level of image fidelity, with the magnitude of the improvement directly related to the fill factor of the data collection.

\section{ACKNOWLEDGEMENTS}

This work has been part of a collaboration between the University of Manchester and Manchester Metropolitan University. The authors wish to thank members of the Centre for Sensing and Imaging at Manchester Metropolitan 
University, Dr. David Andrews for his assistance realizing the initial prototype and Dr. Christopher Johnson for advice about implementing real-time multithreaded C\# applications.

\section{REFERENCES}

[1] Southgate, M.J., "Real Time Spatial Characterisation of Practical Radio Frequency Sources," in MMU Sci. Eng. Conf. (2013).

[2] Southgate, M.J., and Bowring, N.J., "Investigation of a Portable Real Time Radiation Mapper," in CNSDSP14 (2014).

[3] Agurto, A., Li, Y., Tian, G.Y., Bowring, N., and Lockwood, S., "A Review of Concealed Weapon Detection and Research in Perspective,” in Proc IEEE Int. Conf. Netw. Sens. Control, 443-448 (2007).

[4] Salmon, N.A., Kirkham, L., and Wilkinson, P.N., "Characterisation and calibration of a large aperture (1.6 m) kaband indoor passive millimetre wave security screening imager," 2012, 854408-854408-10.

[5] Salmon, N.A., Bowring, N., Hutchinson, S., Southgate, M., and O'Reilly, D., “An aviation security (AVSEC) screening demonstrator for the detection of non-metallic threats at 28-33 GHz," 2013, 890004-890004-7.

[6] Nintendo, "Wii Console Accessories," <https://www.nintendo.co.uk/Wii/Accessories/Accessories-Wii-NintendoUK-626430.html> (20 August 2014).

[7] Peek, B., "Managed Library for Nintendo’s Wiimote,” <http://wiimotelib.codeplex.com/> (1 March 2013).

[8] Taylor, C.T., Hutchinson, S., Salmon, N.A., Wilkinson, P.N., and Cameron, C.D., "Investigation of radio astronomy image processing techniques for use in the passive millimetre-wave security screening environment," 9 June 2014, 90780J.

[9] Högbom, J., "Aperture Synthesis with a Non-Regular Distribution of Interferometer Baselines," Astronomy and Astrophysics Supplement Series 15, 417-426 (1974).

[10] Hutchinson, S., Taylor, C., Fernando, M., Andrews, D.A., and Bowring, N., "Investigation of the CLEAN deconvolution method for use with Late Time Response Analysis of multiple objects," ibid. 